1443

\section{PARENTS PERCEPTION OF MEDICAL VULNERABILITY AND RESILIENCE OF HIS CHILDREN WITH CHRONIC DISEASES}

J.C.S. Herrera ${ }^{1}$, A.M. Treviño², N.H. Domínguez ${ }^{3}$

${ }^{1}$ Pediatric Oncology Unit, ${ }^{2}$ Clinical Laboratory, ${ }^{3}$ Pediatrics Unit, Mexican Social Security Institute, Cd. Obregón, Mexico

Background and aim: Children with chronic diseases can adjust and show profiles of high level resilience and medical vulnerability. Study was directed to know parents perceptions of medical vulnerability and resilience of his children.

Methods: Across convenience sampling was carried out a factorial design study with parents of children's with chronic diseases, attended in a concentration medical unit. Applied in one occasion, a reliable and valid instrument constructed by us, with 67 item's exploring about children's medical vulnerability and resilience parents perception, on agreement with disease impact, death perception, happiness, satisfaction, anxiety and social desire. We established age, gender, disease time and type of treatment groups. Data analysis was non parametric.

Results: Thirty seven parents took part. Leukemia patients predominated (29.72\%). Majority patients had time of disease and treatment six months to 5 years $(72.97 \%$ / $86.48 \%)$. Twenty parents $(54.05 \%)$ considered his children vulnerable and not resilient in $91.89 \%$ (34) cases. Difference existed $(p=0.040)$ among parents in agreement to gender of children only in global punctuation, as well as, between oncologic-cardiac patients parents $(p=0.025)$, oncologic-other chronic conditions $(p=0.025)$, and cardiac-other patients $(p=0.000)$ in vulnerability perception, without in resilience level, also in impact of disease $(p=0.008)$ determined by oncologic parents.

Conclusions: It's possible say that medical vulnerability and resilience not refer necessarily a complementary conditions in the study group. Perception that parents have of his children with chronic disease must be approached in a specific way for group of pathological condition.

Key words: vulnerability, resilience, chronic conditions, special needs.
1444

\section{TRANSPORT OF CHILDREN WITHOUT A STATE STRUCTURED TRANSPORT PROGRAM: THE EXPERIENCE OF AN IRISH CHILDREN'S HOSPITAL}

\author{
K. $\operatorname{Tan}^{1}$, S. Crowe ${ }^{2,3}$
}

${ }^{1}$ Department of Anaesthesia, Intensive Care Medicine \& Pain Management, ${ }^{2}$ Department of Anaesthesia, Intensive Care Medicine and Pain Managament, Adelaide Meath \& National Children's Hospital, ${ }^{3}$ Faculty of Health Sciences, University of Dublin Trinity College, Dublin, Ireland

Objective: To describe the caseload of children presenting to a children's hospital who later required transportation to access pediatric intensive care services.

Design: A retrospective analysis of prospectively collected data.

Setting: A university-affiliated secondary pediatric referral centre with 50 inpatient beds and a three bed high dependency unit.

Subjects: All patients transferred from January $1^{\text {st }}$ 2006, through December 31 2009.

Interventions: None

Measurements and main results: 167 children were transferred over the four year period. The number requiring transport has increased over the described time period. There was a wide variation in the length of time taken to obtain confirmation of the PICU bed availability (71 minutes, range 25-255 minutes in 2009), and the duration of the transport itself. All patients were accompanied by a physician and a registered pediatric nurse. Some children were transferred by two physicians (15\% in 2009), and some by two nurses (37\% in 2009).

Conclusions: Children presenting to a children's healthcare facility which does not provide critical care services may require transfer to another facility. As Ireland does not have a national or state-funded transport system for children, these transfers are carried by staff assigned to other clinical duties. This represents a significant demand on resources in the referring hospital, and is likely to be reflected in the experience of other community pediatric units. 